\title{
Correction
}

Med Klin Intensivmed Notfmed 2019 · 114:45 https://doi.org/10.1007/s00063-017-0363-0

Online publiziert: 24. Oktober 2017

c) Springer Medizin Verlag GmbH 2017

CrossMark

J. Frick' $\cdot$ M. Möckel' $\cdot$ M. Schmiedhofer' $\cdot$ J. Searle' $\cdot$ B. Erdmann² $\cdot$ M. Erhart ${ }^{3}$. A. Slagman'

${ }^{1}$ Arbeitsbereich Notfallmedizin/Rettungsstellen, (CVK, CCM), Charité - Universitätsmedizin Berlin, Campus Virchow-Klinikum, Berlin, Deutschland

${ }^{2}$ Notaufnahme Klinikum Wolfsburg, Wolfsburg, Deutschland

${ }^{3}$ Zentralinstitut für die kassenärztliche Versorgung in Deutschland, Berlin, Deutschland

\section{Correction to: Fragebogen zur Inanspruchnahme der Notaufnahmen}

\section{Implikationen für die Patientenbefragung}

\section{Correction to: \\ Med Klin Intensivmed Notfmed 2017 https://doi.org/10.1007/s00063-017- 0345-2}

In der ursprünglich erschienenen Online-Version des Beitrags wurde der englischsprachige Beitragstitel falsch angegeben.

Korrekt ist: Questionnaire for the utilization of the Emergency Department. Implications for the patient survey.

Der ursprüngliche Artikel wurde aktualisiert.

\section{Korrespondenzadresse}

J. Frick

Arbeitsbereich Notfallmedizin/Rettungsstellen, (CVK, CCM), Charité - Universitätsmedizin Berlin, Campus Virchow-Klinikum Augustenburger Platz 1, 13353 Berlin, Deutschland

johann.frick@charite.de 